\title{
A New Gene Cluster rfe Concerned with the Biosynthesis of Salmonella Lipopolysaccharide
}

\author{
By P. HELENA MÄKELÄ AND M. JAHKOLA \\ State Serum Institute, Helsinki, Finland \\ AND O. LƯDERITZ \\ Max Planck Institut für Immunbiologie, Freiburg, Germany \\ (Accepted for publication 8 October 1969)
}

\begin{abstract}
SUMMARY
A new class of rough mutants has been found in both Salmonella montevi$d e o$ and $S$. minnesota. The mutants resemble phenotypically $r f b$ mutants, having a complete lipopolysaccharide core but no O-specific material in lipopolysaccharide or as free hapten. The site of these $r f e$ mutations is near the isoleucine and valine gene $i l v$, and is thus separate from the known lipopolysaccharide gene clusters $r f a$ for the core, $r f b$ for the O-specific sidechains, and $r f c$ for $\mathrm{O}$ side-chain polymerization. The function of the $r f e$ gene(s) is not known. It is suggested that they might participate in the utilization of the antigen carrier lipid for lipopolysaccharide synthesis, or that they might regulate the activity of $r f b$ genes. The gene determining phosphorylation of the heptose in the lipopolysaccharide core of $S$. minnesota was identified as one within the $r f a$ cluster.
\end{abstract}

\section{INTRODUCTION}

The O-antigenic lipopolysaccharide of Salmonella is thought to have a central core which is common to all Salmonella species. Attached to it in an unknown way are the O side-chains specific for each O-antigenic type (Fig. I). Mutants in which the synthesis of lipopolysaccharide is defective are culturally rough $(\mathrm{R})$. Three classes of rough mutants have been described (Subbaiah \& Stocker, 1964; Naide et al. 1965; Beckmann, Subbaiah \& Stocker, I964). rfa mutants have a defective core, and produce a low molecular weight 'hapten' with $\mathrm{O}$ specificity. The $r f a$ mutations are located in a part of the chromosome near $x y l$ (Fig. 2). Later many, although not all, of the $r f a$ mutations were shown to be cotransducible with pyrE and cysE between $x y l$ and $i l v$ (Kuo \& Stocker, 1968). The hapten is thought to consist of polymerized O-specific repeating units as precursors of $O$ side-chains which cannot be attached to the unfinished core (Kent \& Osborn, I 968). rfb mutants on the other hand have a complete core, but produce no $\mathrm{O}$-specific material. Their mutations map in a tight cluster near his. The third class of mutants, $r f c$, are unable to polymerize the O-specific repeating units. Their sites of mutations are located between trp and gal.

However, some $\mathrm{R}$ mutants do not fit into this scheme. These have a complete core with $N$-acetylglucosamine, and also O-specific hapten (Beckmann, Subbaiah \& Stocker, I964; Gemski \& Stocker, I967), which they apparently cannot attach to the core. Some of these $(r f b T)$ have mutations that map in the $r f b$ cluster. Other mutations 
called $r f a L$ resemble $r f a$ in being cotransducible with pyrE (Kuo \& Stocker, 1968). The LPS core in both cases is also complete in the sense that it can accept $O$ sidechains, when incubated with cell-free preparations of $r f b$ bacteria for example. It is therefore assumed that both $r f b T$ and $r f a L$ mutants might lack a component of a ligase or 'translocase' specified by these genes. Cell envelope preparations of $r f a L$ mutants have been shown to be unable to catalyse the translocation step (Cynkin \& M. J. Osborn, unpublished results quoted by Osborn, 1969).
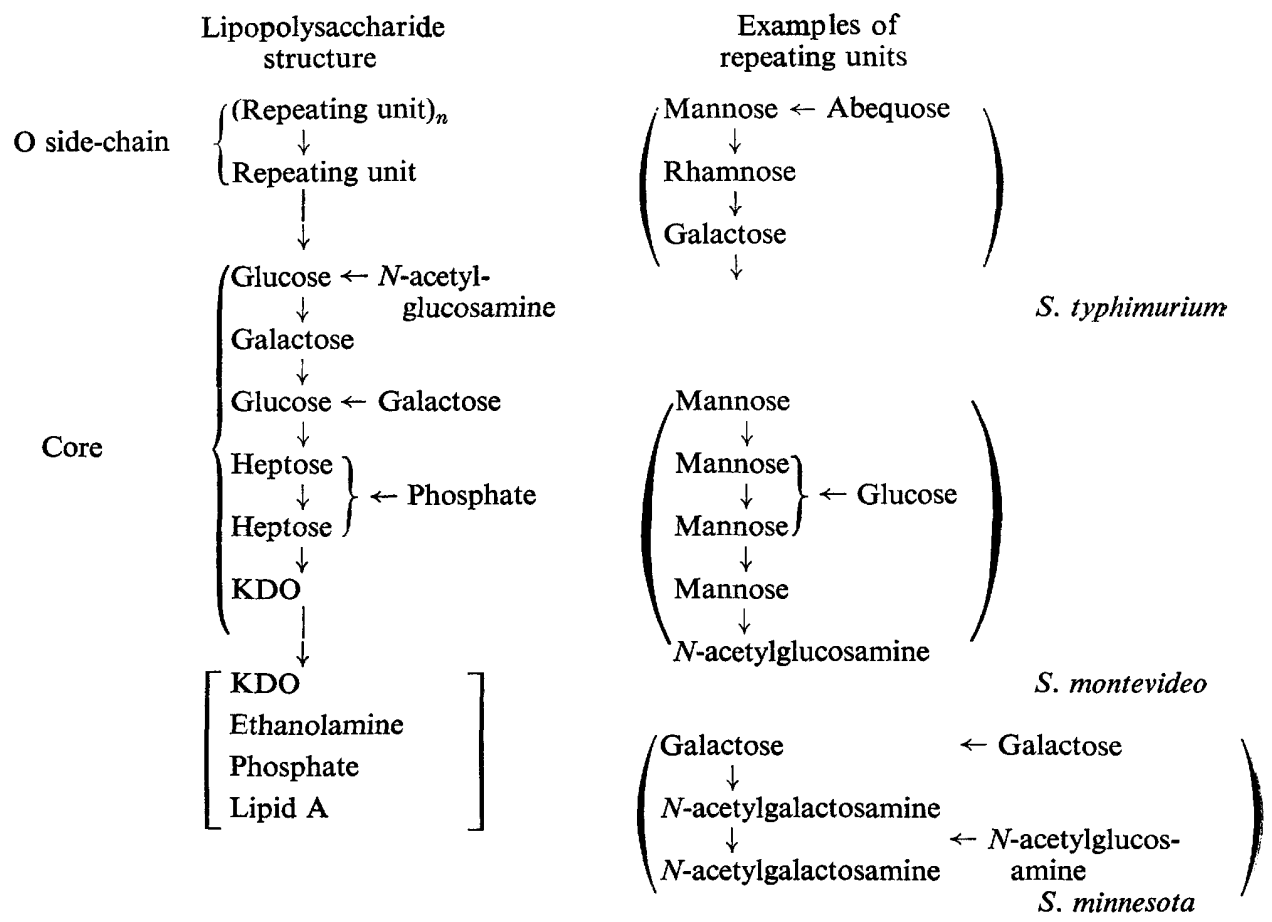

Fig. I. Schematic structure of the Salmonella lipopolysaccharide (Lüderitz, Jann \& Wheat, 1968; Fuller \& Staub, 1968; Osborn, 1969). The innermost part of lipopolysaccharide contains $\mathrm{KDO}$ (= ketodeoxyoctonate), ethanolamine, phosphate and lipid A. To this are attached polysaccharide side-chains composed of a core and an $\mathrm{O}$ side-chain. The $\mathrm{O}$ sidechain is built up of repeating units, which carry the O-specific antigenic determinants characteristic of each Salmonella $O$ group. Examples of the structure of the repeating unit in different Salmonella species, belonging to different $\mathbf{O}$ groups, are shown on the right.

Rough mutants have so far been analysed genetically only in Salmonella typhimurium strain LT2. While studying rough mutants in two other Salmonella species, $S$. montevideo (O antigens 6,7$)$ and $S$. minnesota (O antigen 21 ), we found a new class. of $\mathrm{R}$ mutants ( $r f e$ ) which produce a complete core, but no O-specific hapten. Their sites of mutation are located close to ilv and are clearly separate from $r f a, r f b$ and $r f c$. The symbol $r f e$ is preferred to $r f d$, which would be similar to the rouD used earlier to. describe leaky $r f a$ mutants (Gemski \& Stocker, 1967). Typical $r f a$ mutations in these species, as in S. typhimurium, occur close to $x y l$. 


\section{METHODS}

Bacterial and phage strains. Salmonella typhimurium strain LT 2 and various rough (R) mutants of it were obtained from Dr B. A. D. Stocker, Stanford University, California, as were the O- and R-specific phages (Wilkinson \& Stocker, 1968)

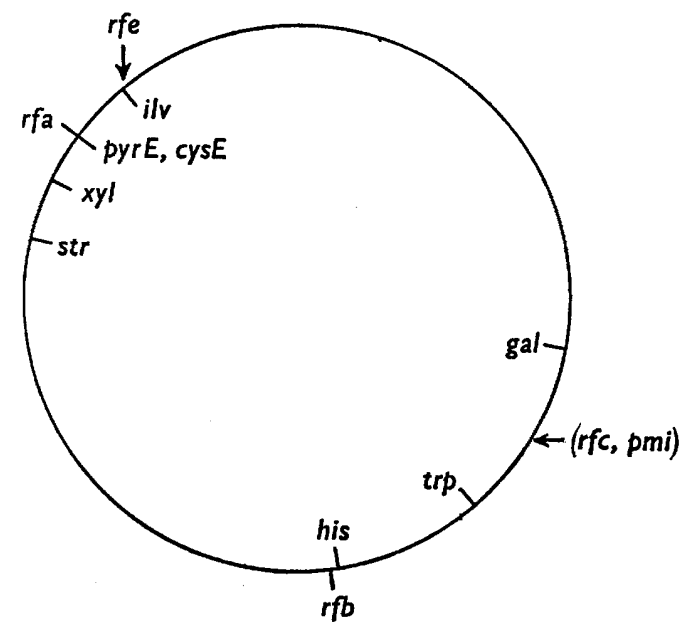

Fig. 2. Part of the chromosome map of Salmonella (after Sanderson, I967; Mäkelä \& Stocker, 1969). Positions not accurately known are indicated by arrows, and loci whose order is not known are bracketed. Genes labelled outside circle affect lipopolysaccharide synthesis. Gene symbols as follows: $g a l=$ galactose utilization, $\operatorname{trp}=$ tryptophan biosynthesis, $h i s=$ histidine biosynthesis, $s t r=$ resistance to streptomycin, $x y l=$ xylose utilization, $p y r E=$ orotidylic acid pyrophosphorylase, cys $E=$ cysteine biosynthesis, ilv = biosynthesis of isoleucine and valine, $p m i=$ phosphomannoseisomerase, $r f a, r f b, r f c, r f e=$ biosynthesis of the $\mathrm{O}$-antigenic lipopolysaccharide.

Salmonella montevideo was strain no. 129 of Edwards \& Bruner (1942), used earlier by Mäkelä (1966); its mutants used are listed in Table I. Salmonella minnesota was strain s99 obtained from Dr F. Kauffmann, State Serum Institute, Copenhagen, Denmark (Lüderitz et al. 1965 ); its R mutants $\mathrm{mR} 3$ and $\mathrm{mR} 592$ were those described by Lüderitz et al. (I966). It turned out that these mutants had more than one $R$ mutation. Single R-mutant derivatives were therefore isolated from them as recombinants in crosses with the smooth parent strain first made $\mathrm{F}^{+}$; they are listed in Table $\mathrm{I}$. Nutritional, fermentation or streptomycin-resistant mutants were selected from these strains by standard procedures (Lederberg, 1950). R mutants were selected as morphologically rough colonies on complete media or as clones resistant to the Felix-or phage. Diethylsulphate was the mutagen used (I0 $\mathrm{mg} . / \mathrm{ml}$. added to an overnight broth culture, and incubation continued for $30 \mathrm{~min}$. at $37^{\circ}$, after which the culture was diluted $\mathrm{r} / \mathrm{ro0}$ in fresh medium and incubated overnight before plating out). The gene symbols are explained in the legend for Fig. 2.

Bacteriological techniques. In the bacterial crosses, $\mathrm{F}^{+}$strains with the $\mathrm{F}$ factor derived from Escherichia coli KI2 (Mäkelä, Lederberg \& Lederberg, I962) were used as donors. The donors were streptomycin-sensitive and streptomycin (I mg./ml.) was added to the selective medium. All $\mathbf{R}$ strains were identified by cultural 


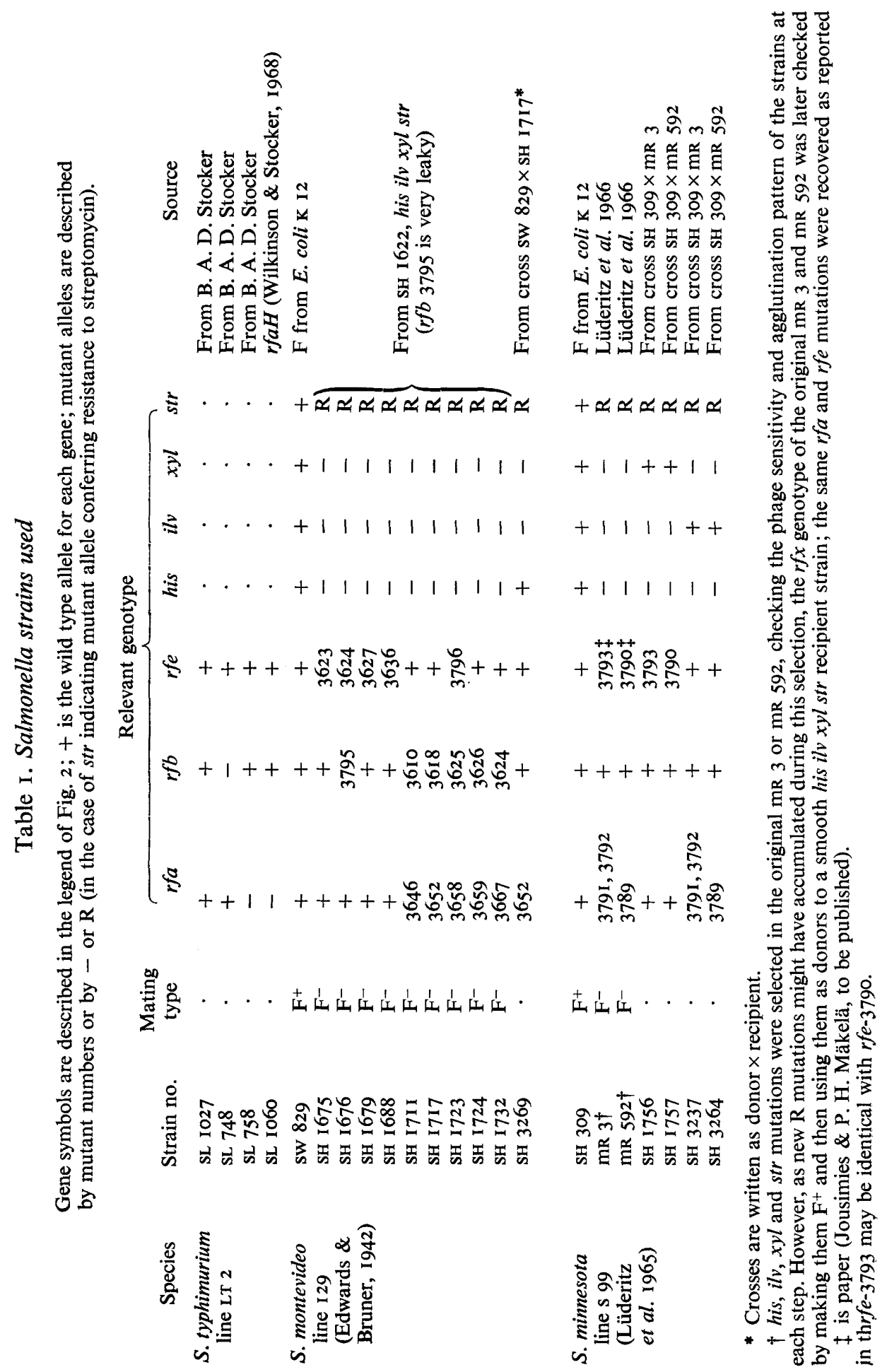


characteristics, slide agglutination ( $4 \%$ saline; anti-O sera diluted in $0.2 \%$ saline), and sensitivity to $\mathrm{O}$ and R-specific phages (Wilkinson \& Stocker, I968). The methods and media were otherwise as described earlier (Mäkelä, I966).

Chemical methods. Lipopolysaccharides were prepared by the phenol-water procedure followed by centrifugation at $105,000 \mathrm{~g}$. Lipopolysaccharide was obtained as the sediment, while the supernatant represents the $L_{1}$ fraction and contains the Ospecific hapten (Beckmann, Subbaiah \& Stocker, 1964). The analyses of these fractions were performed as described previously (Risse et al. 1967). The examination of phosphateless ( $\mathrm{P}-$ ) mutants was performed as described by Dröge, Ruschmann, Lüderitz \& Westphal, 1968). Haemagglutination inhibition by lipopolysaccharide was examined according to Beckmann, Lüderitz \& Westphal (1964).

\section{RESULTS}

\section{Characterization of $R$ mutants with bacteriophages}

Wilkinson \& Stocker (1968) found that the lysis of rough Salmonella typhimurium mutants by selected phages correlated well with the chemical composition of the defective lipopolysaccharide. Some of these phages do not attack S. montevideo or $S$. minnesota at all, but others produce lytic patterns comparable to those in $S$. typhimurium (Table 2). We found sensitivity or resistance to the Felix-oI phage (called FO in this paper) particularly useful. In $S$. typhimurium sensitivity to this phage has been shown to depend on the presence of $N$-acetylglucosamine - that is, the complete core-in the lipopolysaccharide (Lindberg, 1967). Thus smooth bacteria and R mutants that have a complete core are FO-sensitive, while core mutants are FOresistant.

Table 2. Phage sensitivity patterns of smooth and rough strains of Salmonella typhimurium, S. montevideo and S. minnesota

Action of phage (clear lysis $=+$; no lysis $=-$ ) was determined by applying the phage dilutions $\left(\mathrm{IO}^{8}\right.$ plaque-forming units $\left./ \mathrm{ml}\right)$ as drops on nutrient agar plates spread with an overnight broth culture of the bacterial strain and dried for $30 \mathrm{~min}$. (Wilkinson \& Stocker, 1968).

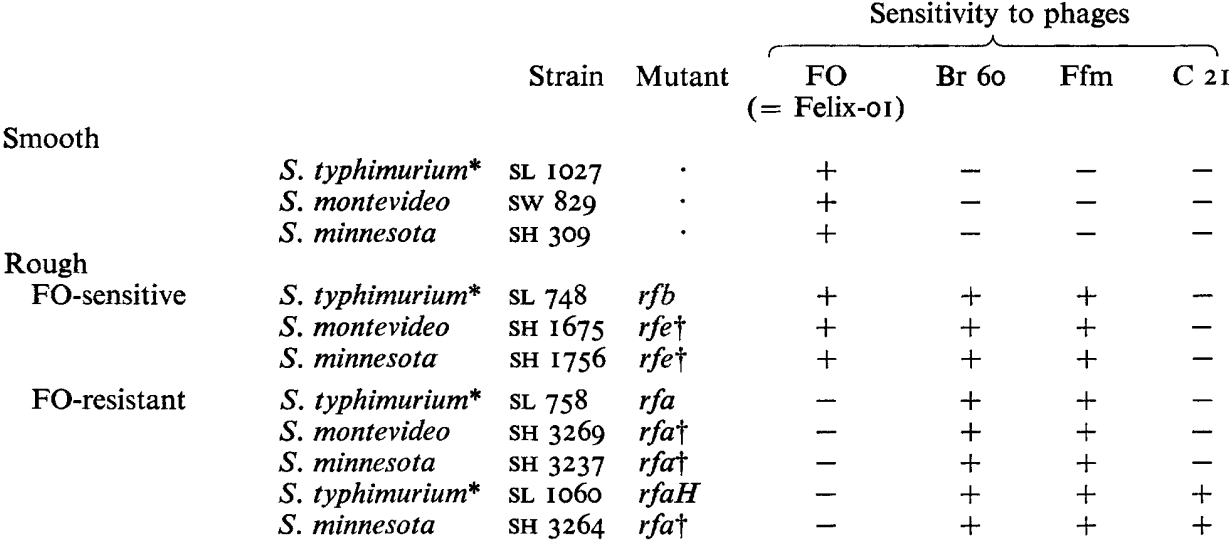

* According to Wilkinson \& Stocker (1968 and personal communication), repeated in this series of experiments.

$\dagger$ Classification according to the data in the present paper. 
(ิ)

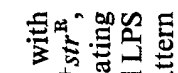

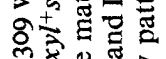

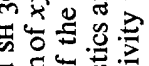

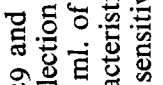

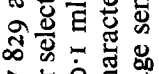

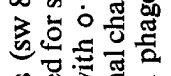

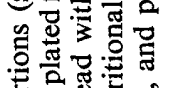

递要寻

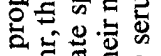

ॐ

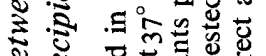

¿ .

ڤ

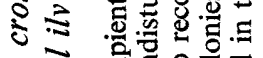

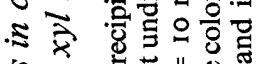

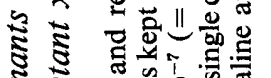

氛

응

రิ

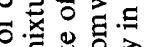

ठे సे है

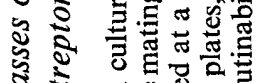

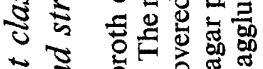

ส

के

을 त्रे

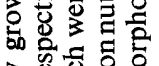

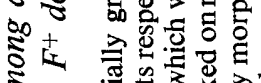

帘

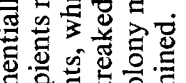

t

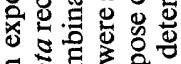

¿े

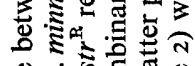

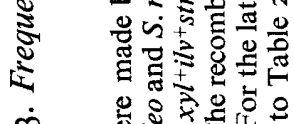

m

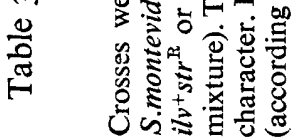

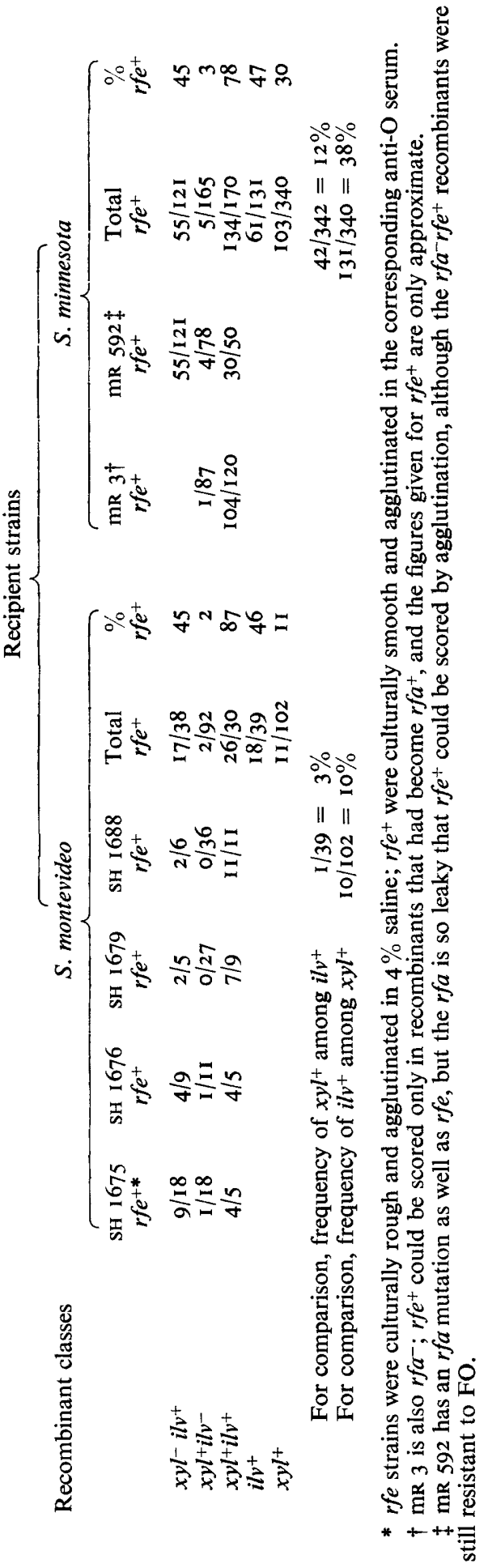




\section{Genetic analysis of rfe mutants}

Most randomly isolated rough mutants of Salmonella montevideo were sensitive to FO and rough-specific phages. Most of these mutations mapped near his, as does $r f b$, but a number did not. All of these mutants whose mutations did not map in the $r f b$ area were isolated from an $x y l i l v$ parent strain which was streptomycin-resistant. To locate the site of these mutations each mutant was crossed with an $\mathrm{F}^{+}$streptomycin-sensitive smooth donor strain of $S$. montevideo (sw 829). The frequency of smooth recombinants among $x y l^{-} i l v^{+}, x y l^{+} i l v^{-}$and $x y l^{+} i l v^{+}$recombinants is given in Table 3. The number of recombinants analysed in each cross is small because of low fertility in $\mathrm{F}^{+}$crosses with this strain. However, the trend was the same in each case: nearly half the $x y l-i l v^{+}$recombinants were smooth, compared with two out of 92 $x y l^{+} i l v^{-}$recombinants. Of the $30 x y l^{+} i l v^{+}$recombinants 26 were smooth, confirming that the $\mathrm{R}$ mutation is in the $x y l-i l v$ region of the chromosome.

In these crosses the unselected $x y l^{+}$donor allele appeared in about $3 \%$ of the $i l v^{+}$ recombinants, and the unselected $i l v^{+}$donor allele in $10 \%$ of the $x y l^{+}$recombinants, as shown in the lower part of Table 3 . The $45 \%$ linkage found between the $\mathrm{R}$ mutation and ilv suggests that this $\mathrm{R}$ mutation is much closer to ilv than is $x y l$. We shall call the gene or genes affected in these mutants $r f e$.

A similar $\mathrm{R}$ mutation was found in two Salmonella minnesota strains. The right half of Table 3 shows some results of mapping this mutation. In both $\mathrm{mR} 3$ and $\mathrm{mR} 592$ the mutation had a similar location to $r f e$ in $S$. montevideo, with $45 \%$ linkage to ilv. The mutants $\mathrm{mR} 3$ and $\mathrm{mR} 592$ were originally isolated by a complex procedure (J. Schlosshardt, personal communication), and have turned out to contain $r f a$ mutations in addition to $r f e$. In MR 592 the $r f a$ mutation was so leaky that $r f e^{+}$could be recognized by smooth colony form and smooth-type agglutination in the corresponding anti-O serum, although the recombinants that were still $r f a^{-}$gave a very slight agglutination in $4 \%$ saline, and retained the FO-resistant phage sensitivity pattern of the $\mathrm{mR} 592$ parent. The $r f a$ in $\mathrm{mR} 3$ was not leaky-the strain has been demonstrated to have two separate biochemical defects in core synthesis (see p. IOI) and hence probably has two independent $r f a$ mutations. With this strain the $r f e$ genotype could be scored only in recombinants that had become $r f a^{+}$. The single $r f e$ mutant derivatives obtained in these crosses from $\mathrm{mR} 3$ and $\mathrm{mR} 592$ as $x y l^{+} r f a^{+}$were phenotypically similar to $r f b$ mutants, e.g. in sensitivity to FO (Table 2, strain SH 1756). Genetically, however, they were very different, as typical his-linked $r f b$ mutants could be easily isolated in the $S$. minnesota strain (unpublished observations). Because of the isolation method of $\mathrm{mR} 3$ and $\mathrm{mR} 592$ we do not know whether the rfe mutation occurred independently in these two strains or not.

\section{Genetic analysis of rfa mutants}

We studied a number of other rough mutants that were resistant to the phage FO. These included five independent mutations of Salmonella montevideo and two of $S$. minnesota. As shown in Table 4, all these mutations mapped closer to $x y l$ than to ilv (34 versus $6 \%$ linkage for the $S$. montevideo mutants, 67 versus $8 \%$ for the $S$. minnesota mutants). The $r \mathrm{fa}^{+}$allele was scored in all recombinants as giving rise to an FO-sensitive phage pattern regardless of the possible presence of an $r f e$ or $r f b$ mutation in the recipients and recombinants. 
Table 3. Frequencies of $r f e^{+}$among different classes of recombinants in crosses between a smooth streptomycin-sensitive $F^{+}$donor and streptomycin-resistant $x y l$ ilv rfe recipients

Crosses were made between exponentially growing broth cultures of donor and recipient, mixed in equal proportions (sw 829 and sH 309 with S.montevideo and S. minnesota recipients respectively). The mating mixture was kept undisturbed at $37^{\circ}$ for $2 \mathrm{hr}$, then plated for selection of $x y l^{+}$str ${ }^{\mathrm{I}}$ $i v^{+} s t r^{R}$ or $x y i^{-} i l v^{+} s t r^{B}$ recombinants, which were recovered at a rate of $c .10^{-7}$ (= Io recombinants per piate spread with $0^{-1} \mathrm{ml}$. of the mating mixture). The recombinants were streaked on nutrient agar plates, from which single colonies were tested for their nutritional characteristics and LPS character. For the latter purpose colony morphology, agglutinability in $4 \%$ saline and in the correct anti-O serum, and phage sensitivity pattern (according to Table 2) were determined.

Recipient strains

Recombinant classes

$x y l^{-} i l v^{+}$
$x y l^{+} i l v^{-}$
$x y l^{+} i l v^{+}$
$i v^{+}$

$x \mathrm{yl}^{+}$

For comparison, frequency of $x y l^{+}$among $i l v^{+}$ For comparison, frequency of $i l^{+}$among $x y l^{+}$

$$
\text { S. montevideo }
$$

$$
\begin{gathered}
\text { sh } 1675 \\
r f e^{+*}
\end{gathered}
$$$$
\text { SH } 1676
$$

$r f e^{+}$

$\mathrm{SH}$
$\mathrm{rfe}$

679

SH 1688
$r f e^{+}$

$4 / 9$
$\mathrm{I} / 11$
$4 / 5$

$2 / 5$
$0 / 27$

$9 / 18$
$I / 18$
$4 / 5$

$2 / 5$

$0 / 27$
$7 / 9$

$2 / 6$
$0 / 36$
II/1 I

$2 / 6$
$0 / 36$
$11 / 11$

$26 / 30$
$18 / 39$
$11 / 102$

$$
\begin{array}{r}
1 / 39=3 \% \\
10 / 102=10 \%
\end{array}
$$

\begin{tabular}{|c|c|c|c|}
\hline $\begin{array}{c}\operatorname{mr} 3^{\dagger} \\
r e^{+}\end{array}$ & $\underset{r f e^{+}}{\operatorname{mr} 592 \ddagger}$ & $\begin{array}{l}\text { Total } \\
r f e^{+}\end{array}$ & $\begin{array}{r}\% \\
r f e^{+}\end{array}$ \\
\hline $\mathrm{I} / 87$ & $\begin{array}{c}55 / 121 \\
4 / 78\end{array}$ & $\begin{array}{r}55 / 12 I \\
5 / 165\end{array}$ & 45 \\
\hline $104 / 120$ & $30 / 50$ & $134 / 170$ & 78 \\
\hline & & $6 I / 13 I$ & 47 \\
\hline
\end{tabular}

S. minnesota

$$
42 / 342=12 \%
$$$$
131 / 340=38 \%
$$

* $r f e$ strains were culturally rough and agglutinated in $4 \%$ saline; $r f e^{+}$were culturally smooth and agglutinated in the corresponding anti- $\mathrm{O}$ serum.

$+\mathrm{mR}_{3}$ is also $r f a^{-} ; r f e^{+}$could be scored only in recombinants that had become $r \mathrm{fa}^{+}$, and the figures given for $r f e^{*}$ are only approximate.

$\ddagger$ mR 592 has an $r f a$ mutation as well as $r f e$, but the $r f a$ is so leaky that $r f e^{++}$could be scored by agglutination, although the $r f a^{-} r f e^{+}$recombinants were still resistant to $\mathrm{FO}$. 


\section{Relative order of the $x y l, r f a$, ilv and rfe genes}

The relative position of the loci $x y l, r f a$, ilv and $r f e$ could be more closely examined in the two Salmonella minnesota mutants $\mathrm{mR} 3$ and $\mathrm{mR} 592$ that have mutations in both $r f a$ and $r f e$ genes. (It is probable that $\mathrm{mR} 3$ has two separate mutations, in two

Table 5. Frequencies of different recombinant classes in crosses between a smooth streptomycin-sensitive $F^{+}$donor and $\mathrm{xyl}^{-} \mathrm{rfa}^{-}$ilv $\mathrm{rfe}^{-} \mathrm{str}^{r}$ recipients of $S$. minnesota

Crosses were made as in Table $3, r f e$ and $r f a$ characterized as in Tables 3 and 4 . Numbers of recombinants belonging to each class are listed in columns 5 and 6 (N.D. = not determined). In the last four columns are given numbers of the recombinants whose genotype could be produced only by four or more cross-overs. (The + after some numbers means that there might be a few more recombinants belonging to these groups which were not identified. Their number is certainly small-less than five-and would affect all columns nearly equally.) Different recombinant classes fall into this category when different orders of the four relevant genes are assumed, permitting conclusions of the most likely gene order. This appears to be the first one, $x y l-r f a-i l v-r f e$, which would necessitate quadruple cross-overs in not more than eleven recombinants.

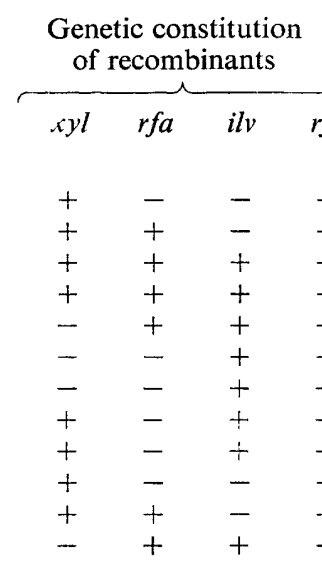

Totals

Recombinants with at least two donor markers (excluding $i l v^{+} r f e^{+}$with the recipient $\mathrm{mR} 3$ )

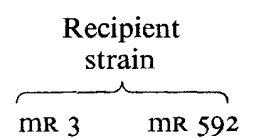

$\begin{array}{rr}\text { N.D. } & 23 \\ 86 & 5 \mathrm{I} \\ \text { I7 } & \text { I9 } \\ \text { I03 } & 28 \\ \text { I } 2 & 8 \\ \text { I67 } & 47 \\ & 62 \\ 3 & \text { I } \\ \text { N.D. } & 2 \\ \text { I } & 2 \\ \text { O } & 2 \\ 389 & 4 \\ & 249\end{array}$

Minimum number of quadruple crossovers required to produce the recombinants observed, assuming gene order

$\overbrace{\begin{array}{cccc}x y l-r f a- & x y l-r f a- & r f a-x y l- & r f a-x y l- \\ i l v-r f e & r f e-i l v & i l v-r f e & r f e-i l v\end{array}}$

\begin{tabular}{ll}
$=$ & - \\
$=$ & 36 \\
$=$ & $\overline{-}$ \\
$=$ & $\overline{-}$ \\
$\overline{6}$ & 6 \\
$2+$ & $2+$ \\
3 & - \\
\hline & 4
\end{tabular}

$\begin{array}{cc}- & - \\ - & 36 \\ \overline{20} & \frac{-}{20} \\ - & - \\ - & - \\ 2+ & - \\ 3 & - \\ 4 & 4 \\ 29+ & 6 \mathrm{I}+\end{array}$

different $r f a$ genes. In this genetic analysis they were never separated, and we feel justified in treating them as a single mutation.) These mutants were crossed with SH 309 (smooth, $\mathrm{F}^{+}$), and all $x y l^{+}$or $i l v^{+}$recombinant classes analysed (Table 5). Taking into account the fairly close linkage between $i l v$ and $r f e$, and between $x y l$ and $r f a$, the four loci can be assigned four different orders as shown in the last four columns of Table 5. Assuming the gene order $x y l-r f a-i l v-r f e$ we see that most of the recombinant classes can be produced through a double cross-over, integrating the donor alleles in the recipient chromosome as a single block. The four classes $x y l^{+} r f a^{-} i l v^{+} r f e^{-}, x y l^{+} r f a^{-} i l v^{+} r f e^{+}, x y l^{+} r f a^{-} i l v^{-} r f e^{+}$and $x y l^{+} r f a^{+} i l v^{-} r f e^{+}$, however, cannot be produced in this simple way but require at least four cross-overs. Eleven representatives of these classes were found among the 386 recombinants that had at least two donor alleles and therefore could contribute to the analysis. Assuming any of the other three gene orders, a larger number of recombinants $(48,29$ and $6 \mathrm{I}$ 


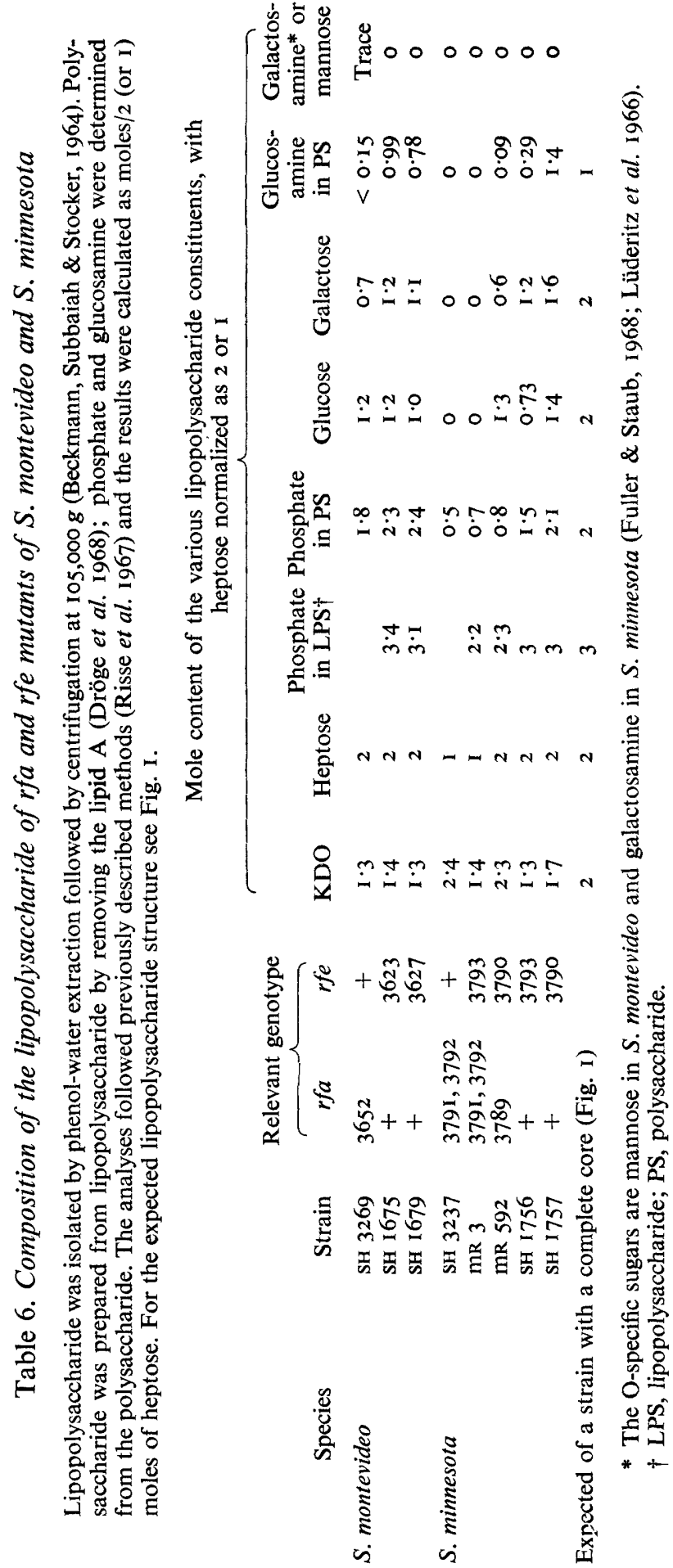


respectively) would require quadruple cross-overs, and therefore the first-mentioned order appears the most likely one.

These results thus locate the rfa cluster of Salmonella minnesota between $x y l$ and $i l v$, as it is in S. typhimurium. To make its homology with the $r f a$ in $S$. typhimurium more certain one would like to test its cotransducibility with, for example, pyrE, which is between $x y l$ and $i l v$ and cotransducible with $r f a$ in $S$. typhimurium. The $r f e$ locus remains clearly separated.

\section{Chemical composition of the lipopolysaccharide and LI fraction of the mutants}

For studying the chemical composition of the mutant lipopolysaccharide we prepared separate $r f a$ or $r f e$ mutant strains from the original double or triple mutants by crossing these with smooth donors of the same species. Thus SH 3269 (Table 6) is a recombinant from the cross between sw 829 as the smooth donor and SH $17 \mathrm{I} 7$ as the recipient; it was selected as his $r f b^{+}$, and has the $r f a-3652$ mutation of SH I7I7. Similarly, a cross between SH 309, the smooth $\mathrm{F}^{+}$Salmonella minnesota strain and mR 3 gave the $r f a$ mutant $\mathrm{sH} 3237$ as an $i l v^{+} r f e^{+}$recombinant, and the $r f e$ mutant SH 1756 as an $x y l^{+} r f a^{+}$recombinant. The other single $r f e$ mutant SH I 757 was prepared as $x y l^{+} r f a^{+}$from a similar cross between SH 309 and MR 592.

The monosaccharide composition of the lipopolysaccharide prepared from these mutants was determined, and the results are given in Table 6. $\mathrm{N}$-acetylglucosamine was analysed from the polysaccharide (PS) obtained from lipopolysaccharide after removal of lipid A (Dröge et al. 1968) because lipid A also contains glucosamine. Phosphate was determined in both lipopolysaccharide and polysaccharide. The molar proportions of these constituents found in strains with the complete lipopolysaccharide core are given as the bottom line of the Table (see also Fig. I, for the structure of the core).

Let us first examine the $r f e$ mutants. Two of these were studied in each species: SH 1675 and SH I679 of Salmonella montevideo and SH I756 and SH 1757 (which may or may not represent the same rfe mutation-see p. 97) of S. minnesota. The sensitivity of these mutants to the phage FO strongly suggests that they contain the com plete lipopolysaccharide core. All the core constituents were indeed found in the lipopolysaccharide prepared from them, and the polysaccharide contained the expected amount of $N$-acetylglucosamine. SH 1756 was the only exception, with a lower value for $N$-acetylglycosamine.

The serological specificity of the $r f e$ lipopolysaccharide was studied by a haemagglutination inhibition technique (Beckmann, Lüderitz \& Westphal, I964). When red blood cells are coated with lipopolysaccharide of various $R$ mutants they become agglutinable by the specific anti- $\mathrm{R}$ serum prepared against this sort of $\mathrm{R}$ lipopolysaccharide; this agglutination can be specifically inhibited by the same sort of lipopolysaccharide but not by others. In this test the $r f e$ lipopolysaccharide could inhibit the haemagglutination by anti-rfaL of cells coated with $r f a L$ lipopolysaccharide and by anti-rfb of cells coated with lipopolysaccharide from several $r f b$ mutants. The $r f e$ lipopolysaccharide thus resembles that of $r f a L$ and $r f b$ mutants, both of which are known to contain the complete core (see Introduction).

Two rfa mutants SH 3269 and SH 3237 were examined. They had no or very little $\mathrm{N}$-acetylglucosamine in the polysaccharide, indicating deficient synthesis of the core. The defect in SH 3269 is apparently leaky, permitting some core stubs to be completed 
and then capped by the O-specific side-chains, as indicated by the presence of some $\mathrm{N}$-acetylglucosamine and traces of $\mathrm{O}$-specific sugars. This strain behaves like a leaky $\mathrm{R}$ mutant also in its reactivity with the specific anti-O serum.

Strain SH 3237 (and its parent mR 3) has a more defective core lacking the second heptose and all other constituents distal to it. Strain mR 3 has been described as a mutant deficient in the transfer of the second heptosyl residue to the first heptose of the core (Risse et al. 1967). Later experience (unpublished observations) has shown that it also lacks the ability to phosphorylate lipopolysaccharide heptose. These two defects probably have their basis in two separate mutations, provisionally designated as $r f a-3791$ for the defect in heptosyl transfer, and $r f a-3792$ for the defect in phosphorylation. In the genetic analysis we have so far been unable to separate the two hypothetical mutations, which might indicate that they represent a deletion encompassing at least parts of two genes; however, no deletion mutations have so far been found in the $r f a$ cluster (Mäkelä \& Stocker, 1969). Strain MR 3 has been shown to possess at least one enzyme participating in core synthesis, namely the transferase for the first glucose residue (Risse et al. 1967 ), which in Salmonella typhimurium is determined by a gene $r f a G$ in the $r f a$ cluster (Wilkinson \& Stocker, I968; Osborn, I968). The hypothetical deletion could thus not extend to this gene. The presence of either two separate mutations or a deletion would also account for the apparent non-leakiness of the defect in core synthesis.

The $r f a-3789$ of the Salmonella minnesota mutant $\mathrm{mR} 592$ was not examined chemically because of its leakiness. $r f a-3789 r f e^{+}$derivatives of $\mathrm{mR} 592$ were nearly smooth in cultural behaviour and $\mathrm{O}$-specific agglutination although they retained a rough FO-resistant phage sensitivity pattern (SH 3264 in Table 2). A conspicuous feature of the $r f a r f e$ double mutant $\mathrm{mR} 592$ itself is the decreased amount of phosphate in both lipopolysaccharide and polysaccharide, indicating a lack of the phosphate normally attached to the core (Table 6). This type of mutant lipopolysaccharide has been described as $\mathrm{P}-$ as opposed to the normal P+ (Dröge et al. 1968). The Plipopolysaccharide is characterized by (I) less phosphate in the lipopolysaccharide and polysaccharide than in $\mathrm{P}+$ strains; (2) no heptosephosphate obtained on hydrolysis of lipopolysaccharide performed according to Slein \& Schnell (I953); and (3) a peak of methylated heptoses obtained after methylation and methanolysis, not seen with $\mathrm{P}+$ lipopolysaccharide. The lack of phosphorylation prevents the completion of the core by preventing the transfer of galactose (Mühlradt, Risse, Lüderitz \& Westphal, I968). The defect in phosphorylation in $\mathrm{mR} 592$ is probably very leaky, as high amounts of glucose and galactose and even some $\mathrm{N}$-acetylglucosamine are found in the lipopolysaccharide and polysaccharide. The absence of $\mathrm{O}$ specific sugars in the lipopolysaccharide preparation is probably accounted for by the rfe mutation in MR 592.

When the $r f a$ of either $\mathrm{mR} 3$ or $\mathrm{mR} 592$ was replaced by the $r f a^{+}$allele, the resulting rfe recombinants SH 1756 and SH 757 had, as shown above, gained the ability to synthesize the complete core. The $\mathbf{P}+$ character of their lipopolysaccharide was specifically confirmed by all the three criteria mentioned above. Thus it has been demonstrated that the phosphorylation of lipopolysaccharide heptoses is determined by a gene belonging to the $r f a$ cluster.

As $r f a$ mutants synthesize an incomplete core, they are unable to attach O-specific side-chains to their lipopolysaccharide. In Salmonella typhimurium rfa mutants the 


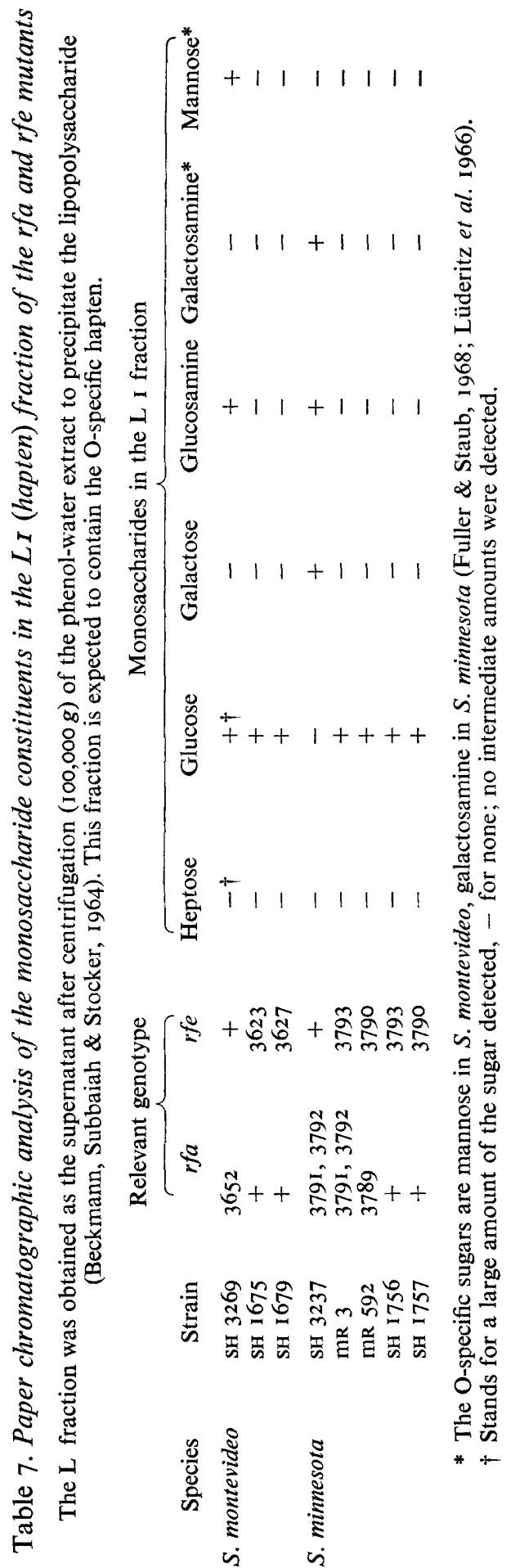


$\mathrm{O}$-specific material is found as haptenic polysaccharide in the so-called $\mathrm{L}_{\mathrm{I}}$ fraction after phenol-water extraction and high-speed centrifugation, which removes lipopolysaccharide (Beckmann, Subbaiah \& Stocker, I964). We isolated the L I fraction from our $S$. montevideo and $S$. minnesota mutants in the same way, and analysed its monosaccharide content by paper chromatography (Table 7). The L I fraction of the $S$. montevideo rfa mutant SH 3269 contained large amounts of glucose, mannose and glucosamine, which are sugars constituting the $\mathrm{O}$-specific side-chains in this species (Fuller \& Staub, 1968). Of these sugars mannose is 'O-specific', occurring only in the $\mathrm{O}$ side-chains in the bacterium. Similarly the $r f a$ mutant SH 3237 of $S$. minnesota contained in its L I fraction galactose, glucosamine and galactosamine, which are the constituents of its O side-chains (Lüderitz, Jann \& Wheat, 1968), and of which galactosamine is O-specific. None of the four $r f e$ mutants contained O-specific sugars in the $L_{I}$ fraction. Glucose was found in most of the $L_{I}$ fractions; it probably derives from cell-wall glucans. The original $S$. minnesota mutants $\mathrm{mR} 3$ and $\mathrm{mR} 592$ have no hapten no doubt because of their $r f e$ mutation-when the $r f e$ was replaced by the $r f e+$ allele (to obtain SH 3237 from $\mathrm{mR} 3$ ), the strain gained the ability to synthesize hapten as expected of an $r f a$ mutant.

\section{DISCUSSION}

Mutations at two separate locations in the $x y l$, ilv region of Salmonella montevideo and $S$. minnesota resulting in defects in the synthesis of lipopolysaccharide were demonstrated. One group corresponds to the rfa gene cluster described in S. typhimurium, which determines the synthesis of the lipopolysaccharide core, including its phosphorylation. The other group, the rfe mutants, has not been detected in $S$. typhimurium, and its function is unknown. The rfe mutants resemble phenotypically the $r f b$ and $r f a L$ mutants of $S$. typhimurium. All of these are sensitive to FO and several rough-specific phages, and they all synthesize a core lipopolysaccharide with $\mathrm{N}$-acetylglucosamine. The $r f a L$ mutants contain O-specific hapten and the $r f a L$ gene is apparently concerned with the translocation of the $\mathrm{O}$ side-chain from its lipid carrier to the lipopolysaccharide core (Osborn, 1969). The $r f b$ mutants are defective in the synthesis of $\mathrm{O}$-specific sugars or in their assembly into the $\mathrm{O}$ side-chains, and therefore have no hapten (Beckmann, Subbaiah \& Stocker, 1964; Nikaido, Nikaido, Subbaiah \& Stocker, 1964; Nikaido, Levinthal, Nikaido \& Nakane, 1967).

The rfe mutants of Salmonella montevideo and $S$. minnesota synthesize no hapten either (Table 7), and therefore it seems most likely that their mutation interferes with the biosynthesis of $\mathrm{O}$-specific material. The $r f b$ cluster is generally known to harbour most of the genes whose products participate in the synthesis of O-specific repeating units (Stocker, Wilkinson \& Mäkelä, I966), both the genes for the synthesis of the O-specific monosaccharides and the genes for the transferases assembling the repeating units. This was specifically shown to be true of the $r f b$ cluster of $S$. montevideo. When the $r f b$ region of the $S$. montevideo chromosome was introduced, by conjugation, into the cells of $S$. typhimurium, these started synthesizing a lipopolysaccharide of the S. montevideo type (Mäkelä, I966; Nikaido, Nikaido \& Mäkelä, I966). It was concluded that all the information necessary for the synthesis of $S$. montevideo-specific lipopolysaccharide, and not shared by $S$. typhimurium, was located in the $r f b$ gene cluster. Thus if the $r f e$ gene(s), remote from $r f b$, specify a product necessary for the 
synthesis of O side-chains, this function must be common to $S$. montevideo and $S$. typhimurium. What could such a common function be? The only monosaccharide found in the $\mathrm{O}$ side-chains of both these species is mannose (Fig. I). However, the enzymes participating in the synthesis of mannose (as its guanosine-diphosphate nucleotide) are specified by $r f b$ genes, or by a gene $p m i$ (for phosphomannoseisomerase) between $t r p$ and $g a l$ (Stocker, Wilkinson \& Mäkelä, I966; Nikaido et al. 1967). Furthermore, mannose is not present in the O side-chains of S. minnesota, in which rfe gene(s) were also identified. The antigen carrier lipid (Wright, Dankert \& Robbins, 1965) on which the $O$ side-chains are assembled, is very likely to be common to all Salmonella species. The possible role of $r f e$ in antigen carrier lipid synthesis now seems to deserve serious investigation. There are, however, immediate objections to this hypothesis: a similar antigen carrier lipid is involved in the synthesis of the cell-wall peptidoglycan (Higashi, Strominger \& Sweeley, 1967). Bacteria with an impaired synthesis of peptidoglycan would not be viable under the normal growth conditions used in the isolation and study of all our $\mathrm{R}$ mutants. Therefore we might rather think that the antigen carrier lipid will have to be modified in a certain way to mark it for either peptidoglycan or lipopolysaccharide (or some other) synthesis. This modification could be a slight chemical change or perhaps rather a fixed localization of the lipid in association with the enzymes participating in the relevant synthesis. On the other hand, the $r f e$ gene(s) might have a regulatory function, e.g. for the $r f b$ genes. So far no genetic regulation of the $r f b$ genes has been found, and the $r f b$-determined enzymes have shown the same levels of activity whatever the growth conditions of the bacteria (Nikaido, I968).

The work in Helsinki was supported by the Finnish Medical Research Council and by U.S. Public Health Service grant GM-I2046. The assistance of Miss Marianne Hovi is gratefully acknowledged. One of us (O.L.) thanks the Deutsche Forschungs.

\section{REFERENCES}

Beckmann, I., Lüderitz, O. \& Westphal, O. (I964). Zur Immunchemie der somatischen Antigene von Enterobacteriaceae. IX. Serologische Typisierung von Salmonella-R-Antigenen. Biochemische Zeitschrift 339, $40 \mathrm{I}$.

Beckmann, I., Subbaiah, T. V. \& Stocker, B. A. D. (1964). Rough mutants of Salmonella typhimurium. 2. Serological and chemical investigations. Nature, London 20r, I 299.

Dröge, W., Ruschmann, E., Lüderitz, O. \& Westrhal, O. (I968). Biochemical studies on lipopolysaccharides of Salmonella R mutants. 4. Phosphate groups linked to heptose units and their absence in some R lipopolysaccharides. European Journal of Biochemistry 4, I34.

Edwards, P. R. \& Bruner, D. W. (1942). Serological identification of Salmonella cultures. Circular. Kentucky Agricultural Experiment Station 54.

Fuller, N. A. \& Staub, A. M. (1968). Immunochemical studies on Salmonella I3. Chemical changes appearing on the specific polysaccharide of $S$. cholerae suis $\left(6_{2}, 7\right)$ after its conversion by phage I4 $(6,7)$. European Journal of Biochemistry 4, 286.

Gemski, P. JuN. \& STOCKeR, B. A. D. (I967). Transduction by bacteriophage P22 in non-smooth mutants of Salmonella typhimurium. Journal of Bacteriology 93, 1588.

Higashi, Y., Strominger, J. L. \& Sweeley, C. C. (I967). Structure of a lipid intermediate in cell wall peptidoglycan synthesis: a derivative o fa $\mathrm{C}_{55}$ isoprenoid alcohol. Proceedings of the National Academy of Sciences of the United States of America 57, 1878.

Kent, J. L. \& OsBorn, M. J. (1968). Haptenic O-antigen as a polymeric intermediate of in vivo synthesis of lipopolysaccharide by Salmonella typhimurium. Biochemistry, New York 7, 4419.

Kuo, T. T. \& STOCKER, B. A. D. (1968). Transduction of $r f a$ (rouA) genes of Salmonella typhimurium strain LT 2 by phage ESI 8. Bacteriological Proceedings p. 56. 
LEDERBERG, J. (1950). Isolation and characterization of biochemical mutants of bacteria. Methods in Medical Research 3, 5.

LinDBERG, A. A. (1967). Studies of a receptor for Felix O-I phage in Salmonella minnesota. Journal of General Microbiology 48, 225.

Lüderitz, O., Galanos, C., Risse, H. J., Ruschmann, E., Schlecht, S., Schmidt, G., SchulteHolthausen, H., Wheat, R., Westphal, O. \& Schlosshardt, J. (I966). Structural relationships of Salmonella $\mathrm{O}$ and R antigens. Annals of the New York Academy of Sciences 133, 349.

LÜDERITZ, O., JANN, K. \& WhEAT, R. (1968). Somatic and capsular antigens of Gram-negative bacteria. In Comprehensive Biochemistry. Ed. by M. Florkin and E. H. Stotz, vol. 26 A, p. 105.

Lüderitz, O., Risse, H. J., Schulte-Holthausen, H., Strominger, J. L., Sutherland, I. W. \& WESTPHAL, O. (1965). Biochemical studies of the smooth-rough mutation in Salmonella minnesota. Journal of Bacteriology 89, 343.

MÄKELÄ, P. H. (1966). Genetic determination of the O antigens of Salmonella groups B $(4,5,12)$ and C $(6,7)$. Journal of Bacteriology 9I, II 15.

MÄKelä, P. H., Lederberg, J. \& Lederberg, E. M. (I962). Patterns of sexual recombination in enteric bacteria. Genetics $47,1427$.

MÄKeLё, P. H. \& STOCKER, B. A. D. (1969). Genetics of polysaccharide biosynthesis. Annual Review of Genetics 3, 29I.

MühlRadt, P., Risse, H. J., LÜDeRitz, O. \& WestPhal, O. (I968). Biochemical studies on lipopolysaccharides of Salmonella R mutants. 5. Evidence for a phosphorylating enzyme in lipopolysaccharide biosynthesis. European Journal of Biochemistry 4, I 39.

Naide, Y., Nikaido, H., MÄkelä, P. H., Wilkinson, R. G. \& Stocker, B. A. D. (1965). Semirough strains of Salmonella. Proceedings of the National Academy of Sciences of the United States of America 53, 147.

NIKaIDo, H. (1968). Biosynthesis of cell wall lipopolysaccharide in Gram-negative bacteria. Advances in Enzymology 31, 77.

Nikaido, H., Levinthal, M., Nikaido, K. \& Nakane, K. (1967). Extended deletions in the histidinerough-B region of the Salmonella chromosome. Proceedings of the National Academy of Sciences of the United States of America 57, 1825.

NiKaIdo, H., NiKaido, K. \& MÄKelä, P. H. (1966). Genetic determination of enzymes synthesizing O-specific sugars of Salmonella lipopolysaccharides. Journal of Bacteriology 91, 1126.

Nikaido, H., Nikaido, K., Subbaiah, T. V. \& Stocker, B. A. D. (I964). Rough mutants of Salmonella typhimurium. 3. Enzymatic synthesis of nucleotide-sugar compounds. Nature, London 2or, I 301.

OsBorn, M. J. (1968). Biochemical characterization of mutants of Salmonella typhimurium lacking glycosyl or galactosyl lipopolysaccharide transferases. Nature, London 217, 957.

Osborn, M. J. (1969). Structure and biosynthesis of the bacterial cell wall. Annual Review of Biochemistry 38, $50 \mathrm{I}$.

Risse, H. J., Dröge, W., Ruschmann, E., Lüderitz, O., WestPhal, O. \& Schlosshardt, J. (1967). Eine neue Gruppe von Salmonella R-Mutanten. Serologische und biochemische Analyse des Heptosekerns von Lipopolysacchariden aus Salmonella minnesota und Salmonella ruiru Mutanten. European Journal of Biochemistry $\mathbf{1}, 2 \mathrm{I} 6$.

SANDERSON, K. E. (1967). Revised linkage map of Salmonella typhimurium. Bacteriological Reviews 3I, 354 .

SLeIN, M. W. \& SCHNELl, G. W. (1953). An aldoheptose phosphate in a polysaccharide isolated from Shigella flexneri. Proceedings of the Society for Experimental Biology and Medicine 82, 734.

Stocker, B. A. D., Wilkinson, R. G. \& MäKel̈̈, P. H. (1966). Genetic aspects of biosynthesis and structure of Salmonella somatic polysaccharide. Annals of the New York Academy of Sciences 133, 334

Subbaiah, T. V. \& Stocker, B. A. D. (1964). Rough mutants of Salmonella typhimurium. I. Genetics. Nature, London 201, I 298.

Wilkinson, R. G. \& STOCKER, B. A. D. (1968). Genetics and cultural properties of mutants of Salmonella typhimurium lacking glucosyl or galactosyl lipopolysaccharide transferases. Nature, London 217, 955.

Wright, A., DANKERT, M. \& RoBbins, P. W. (1965). Evidence for an intermediate stage in the biosynthesis of the Salmonella O-antigen. Proceedings of the National Academy of Sciences of the United States of America 54, 235. 